\title{
Surveillance of the Buruli Ulcer in The Democratic Republic of Congo (Drc): Preliminary Results (2016-2018)
}

\author{
Marie José Kabedi Bajani*1, Mintsey $\mathbf{N}^{1}$, Nkunku L ${ }^{1}$, Lunguya $0^{1}$, Kimbonza $S^{1}$, Eddyani $\mathbf{M}^{2}$, Jong \\ $\mathrm{B}^{2}$, Taba $\mathrm{K}^{3}$ and Muyembe $\mathrm{JJ}^{1}$ \\ ${ }^{1}$ National Institute for Biomedical Research (INRB), University of Kinshasa, Democratic Republic of the Congo \\ ${ }^{2}$ Public Health, Institute of Tropical Medicine, Belgium.
}

${ }^{3}$ Department of Chemistry Organic, University of Kinshasa, Democratic Republic of the Congo

*Corresponding author: Marie José Kabedi Bajani, National Institute for Biomedical Research (INRB), University of Kinshasa, Democratic Republic of the Congo Email: bedye2001@yahoo.fr

To Cite This Article: Marie José Kabedi Bajani, Surveillance of the Buruli Ulcer in The Democratic Republic of Congo (Drc): Preliminary Results (2016-2018). Am J Biomed Sci \& Res. 2019 - 6(5). AJBSR.MS.ID.001062. DOI: 10.34297/AJBSR.2019.06.001062.

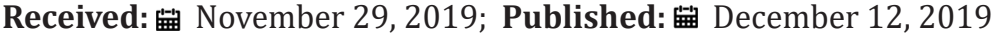

\section{Abstract}

Context: Buruli ulcer poses a real public health problem, not well known, calling for international mobilization. It is an emerging threat to public health in many rural and inter-tropical regions, and DRC is not immune to this problem. However, improving the visibility of this disease and mobilizing resources are ways to make disease control activities more effective.

Objective: To gather information on Buruli ulcer in the different provinces of the DRC. Material and methods: Samples came from different provinces of DRC from patients uspected of Buruli ulcer. Two techniques were performed namely Ziehl-Nelsen and real-time PCR to confirm cases. Data comparison was performed using the Chi-square test.

Results: During 3 years of surveillance (2016-2018), 984 samples were recorded, of which $86.1 \%$ were swabs. 72 (7.3\%) were Ziehl positive and $251(25.51 \%)$ were confirmed by PCR- $(\mathrm{p}<0.001)$. However, the positivity by province, led by Equateur $(11 / 19,57.9 \%)$, followed by Kongo Central $(215 / 707,30.4 \%)$ and Maniema (16/52, 30, 7\%). On the other hand Haut Uele and Kinshasa had respectively 3/13(23, 1\%) and 13/188(6, 9\%).

Conclusion: Buruli ulcer surveillance is a public health goal both to measure the importance and trends of its incidence in DRC. This monitoring is important for better documenting the burden of the disease, mapping endemic villages and also ensuring better case management.

Keywords: Surveillance; Buruli Ulcer; DRC; Results; preliminaries

\section{Introduction}

Buruli ulcer (BU) poses a real public health problem that is not well known, calling for international mobilization [1-3]. It is an emerging threat to public health in many rural, inter-tropical regions [1-3]. The infection often leads to extensive destruction of the skin and soft tissues, with extensive ulceration usually on the limbs and may result in permanent deformity and disability [1-4]. According to partial data from 13 countries, there were 2206 cases in 2017 against 1920 in 2016 as reported by the global report of the World Health Organization (WHO) [1-3]. In Africa, about 48\% of the affected population are children under 15, while in Australia, they account for only $10 \%$ of cases and 19\% in Japan [1-3].

The Democratic Republic of Congo is concerned by this emerging threat to public health. According to the 2014 mapping, there are 17 endemic health zones (3\%), 26 are suspected of being endemic (5\%) and 472 are non-endemic (92\%) [4]. The disease is still largely unknown in DRC and is often considered a marginal disease. However, most cases are reported in Kongo 
Central Province [4-7]. Thus, early diagnosis and treatment are the only means to minimize morbidity and avoid long-term disability [1-3]. It is within this framework that the National Institute for Biomedical Research (INRB) has set up a surveillance system for the confirmation of cases by molecular test.

\section{Objective}

To confirm cases and document Buruli ulcer in the different provinces of DRC.

\section{Sources and Methods}

INRB relies on the various health zones of DRC to carry out surveillance of BU.

\section{Sample Collection and Processing Sites}

Samples of BU suspects from different provinces of DRC (Kinshasa, Equateur, Haut Uele, Bas Uele, Maniema and Kongo Central) were included in the study. These samples were registered in the INRB mycobacteria laboratory from January 1, 2016 to December 31, 2018. Samples were analyzed by Ziehl-Nelsen and real-time PCR for case confirmation.

\section{Data Processing}

The data were recorded in the laboratory register and then transferred to the Excel software. Data comparison was done using the Chi-square test and the significance level was set at $5 \%$ and the 95\% confidence interval (CI).

\section{Results}

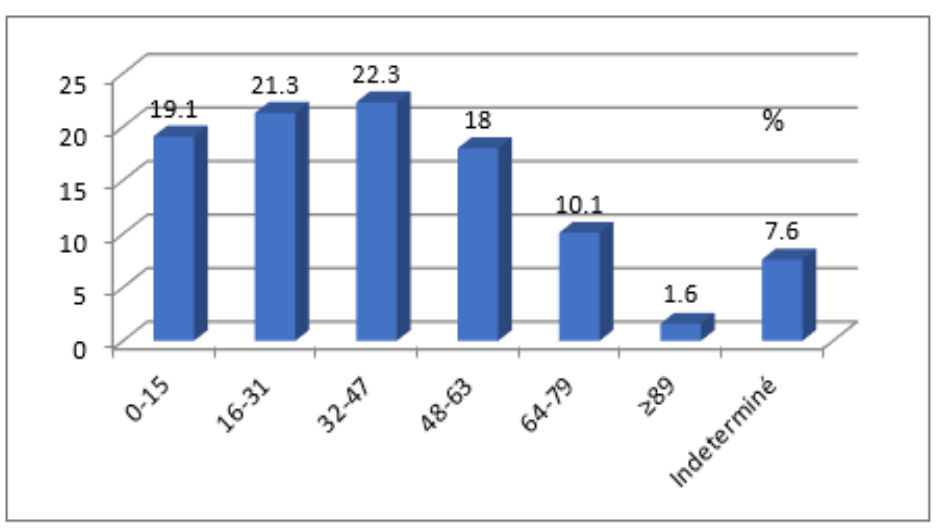

Figure 1: Distribution of samples by age group of patients.

During the three years of surveillance (2016-2018), 984 samples from Buruli ulcer suspects were recorded. Seven hundred and seven samples were from Kongo Central (71.85\%), 188 from Kinshasa (19.1\%), 52 from Maniema (5.3\%), 19 from Equateur (1.9\%), 13 from Haut Uélé (1.3\%) and 5 from Bas Uélé (0.5\%).
$43.9 \%$ of the samples were from female patients, $50.6 \%$ from male, and for $5.5 \%$ of patients ( $p=0.002$ ) gender was not determined. The sex ratio was 1.2. The average age of patients was 36.75 years with extremes ranging from 1 month to 89 years. The distribution by age group is shown in (Figure 1). 


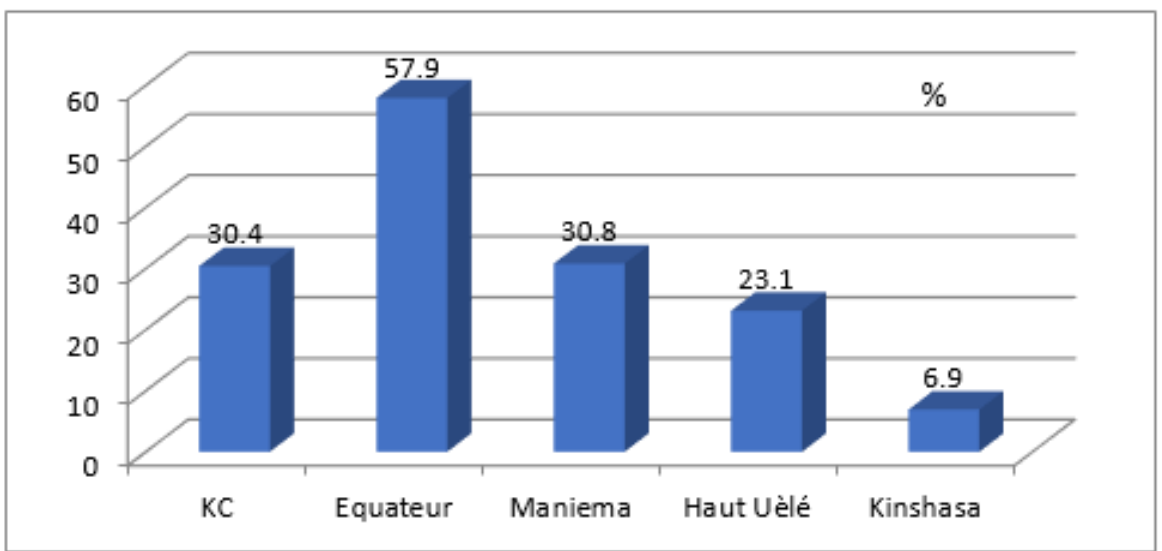

Figure 3: Positivity by province

As shown in figure 1, 22.3\% of samples came from the $32-47$ age group followed by $21.3 \%$ from the $16-31$ age brackets. The $0-15$ and 48-63 age groups had almost the same proportions (19.1\% and $18 \%)$. The distribution of samples according to the type of sampling showed that $86.1 \%$ were swabs, $11.5 \%$ were fine needle samples, $1.3 \%$ were biopsies and $1.1 \%$ were suspensions. After analysis, the overall positivity was $26.2 \%$ (258). This positivity by test is shown in (Figure 2) Figure 2 shows that the proportion of Ziehl positive was $7.3 \%$ and $25.5 \%$ were confirmed by PCR; $\mathrm{p}=0.000$. The positivity by province is shown in (Figure 3). As can be seen in figure 3, Equateur came in first (57.9\%), followed by Maniema (30.8\%) and Kongo Central (30.4\%). On the other hand, Haut Uélé and Kinshasa had respectively $23.1 \%$ and $6.9 \%$.

\section{Discussion}

Surveillance of BU is of paramount importance, as people affected with the disease can end up with more or less significant disabilities for life [1-10]. And so, confirmation of cases by molecular testing appears both as a necessity and a priority [110]. The purpose of the surveillance is to confirm cases and to document the BU in suspected provinces in order to allow a better case management. This surveillance during the three years shows essentially an overall proportion of $26.2 \%$ PCR positive samples and in Ziehl - Nelsen, the proportion was low (7.2\%). These results corroborate those in the literature which reports that the ZN is less sensitive [1-3].

The reported case confirmation shows that Buruli ulcer is a growing threat in DRC and this requires good surveillance of the disease for better determination of the extent of the problem in the country and also ensure good case management in order to avoid complications. However, the proportion of $26.2 \%$ reported in this series is lower than those of $35.9 \%$ and $75 \%$, observed respectively by $[5,7]$ but it is higher than that obtained by [6]. The difference in frequency can be explained by the size of the sample $(97,24$ and 175). The distribution of samples by province puts Kongo Central in the lead $(71.85 \%)$ and this is justified by the fact that the activities of the fight against the disease are well coordinated in this province than in the others in DRC.

The positivity of the samples was very high in Equateur compared to the others provinces which also sent samples for analysis. This can probably be explained by the higher risk of exposure of populations in contact with inappropriate water point in the region and the presence of swampy wetlands [9-12]. Therefore, the hydrographic factor appears to be the determining factor in the outbreak of the disease in this region [9-12]. he City of Kinshasa also has marshy parts and we had confirmed the cases although the proportion of positive samples is low (6.9\%). That is why we must impose and sustain the campaigns of information and sensitization of communities at all levels to help patients to consult in time and avoid the worst because traditional knowledge of the disease remains dominant and linked to a bad curse.

Our results are in agreement with literature data that report the proximity of streams, swamps, and natural or artificial lakes near Buruli ulcer foci (1.8-11). Both sexes were equally affected with a sex ratio of 1.2 in favor of men. The preponderance of the male sex $(50.6 \%)$ is statistically significant $(p=0.002)$ in this survey, but does not agree with the majority of authors who think that the woman would be more exposed although the difference is not more pronounced. in relation to the man given his daily activities [1]. This is the case of [12] who showed in their study that the majority of patients were women (66.7\%) [12]. On the other hand, other studies do not reveal significant differences between men and women [13-15]. For the age group 0-15 years, the proportion reported in this study (19.1\%) agree with those of $17.6 \%, 10 \%$ and $19 \%$ found respectively in DRC, Australia and Japan (6.1). But, we note that our proportion is small compared to $39.8 \%$ reported by Yeboah in Ghana [13].

Although the proportion of attacks is low in this age group in our study, we need to sustain the awareness, training and education of the Congolese population given the chronic evolution and the disabling nature of the disease; disease strike of children leads to 
a double social problem of school drop-out and reintegration to be solved by the community [14]. Regarding samples analyzed in the laboratory, $86.1 \%$ were swabs. This is related to the advanced stage of the disease where we find more ulcerations of different dimensions [5,10-16]. Our results are in agreement with those in the literature which show that in many countries where Buruli ulcer is endemic, 70 to $100 \%$ of patients present with ulcerative lesions and 0 to $30 \%$ with non-ulcerous lesions [1-2] have also reported in their series a predominance of ulcerous lesions [5,6,15-16].

\section{Conclusion}

Confirmation of cases using laboratory methods, gene amplification (PCR) or direct examination of smears, is an essential aspect in the overall management of the disease because the results obtained can guide a set of short-term and medium-term actions to limit the adverse effects of the disease on populations.

\section{Conflict of interest}

None.

\section{References}

1. Aubry P, Gauzere BA (2017) Ulcère de Buruli. Médecine tropicale.

2. WHO (2001) Diagnostic de l'infection à Mycobacterium ulcerans. Who/ CDS/CPE/GBUI Geneva, Switzerland.

3. WHO (2004) Guidedes techniquesde prélèvement d'échantillons pour la confirmation en laboratoire de l'infection à Mycobacterium ulcerans, Who/CDS/CPE/ GBUI?

4. Kibadi K, Tiendrebeogo A, Eboue Kinvi B, B De Jong, M Boelaert, et al. (2014) Ulcère de Buruli dans les zones de santé en République démocratique du Congo, de 1950 à 2013: revue documentaire et nouvelle carte de distribution. Médecine et Santé Tropicales 24: 420-429.

5. Marie José Kabedi Bajani, Nadine Mintsey Mi-Makuth, Octavie Lunguya Metila, Léontine Nkuku Ataraba, Servet Kimbonza Sumba, et al. (2019)
Performance of the Sodium Acetate Method in the Extraction of Mycobacterium ulcerans DNA. IJISR 8(2): 1367-1371.

6. Busakayi CY (2016) Ulcère de Buruli dans la Ville province de Kinshasa: résultats des analyses microbiologiques à l'INRB de Janvier 2011-Mai 2016. Annales Africaines de Médecine 10(1).

7. Phanzu MD (2009) Contribution à l'amélioration du contrôle de l'ulcère de Buruli dans le territoire de Songololo, en République Démocratique du Congo.Th.Méd.

8. Johnson PDR, Veitch MGK, Leslie DE, Flood PE, Hayman JA, et al. (1996) The emergence of Mycobacterium ulcerans infection near Melbourne. Med J Austr 164(2): 76-78.

9. Oluwasanmi JO, Solanke TF, Olurin EO, Itayemi SO, Alabigo, et al. (1976) Mycobacterium ulcerans (Buruli) skin ulcerationin Nigeria. Am J Trop Med Hyg, 25(1): 122-128.

10. Portaels $F$ (1989) Epidémiologie des ulcères à Mycobacterium ulcerans. Ann Soc Belge Méd Trop 69: 91-103.

11.Ziefer AM, Connor DH, Gibson DW (1981) Mycobacterium ulcerans: Infection of two patients in Liberia. Int J Dermatol 20(5): 362-367.

12. Ukwaja KN, Meka AO, A de Chukwuka, Asiedu KB, Huber KL, et al. (2016) Buruli ulcer in Nigeria: results of a pilot case study in three rural districts. Infect Dis Poverty 5:39.

13. Yeboah-Manu D, Aboagye SY, Asare P, Asante-Poku A, Ampah K, et al. (2018) Laboratory Confirmation en laboratoire des cas d'ulcère de Buruli au Ghana, 2008-2016. PLoS Negl Trop 12(6): e0006560.

14. Marston BJ, Diallo MO, Horsburgh CR, Diomande I, Saki MZ, et al. (1995) Emergence of Buruli ulcer disease in Daloa region of Côte d'Ivoire. Am J Trop Med Hyg 52: 219-224.

15. Herbinger KH, Adei O Awua NY, Boateng, Bretzel G (2009) Comparative study of sensitivity of diagnostic methods for the laboratory diagnosis of Buruli ulcer disease.Clin Infect Dis 48(8): 1055-1064.

16. Ayelo GA, Anagonou E, Wadagni AC, Barogui YT, Dossou AD, et al. (2018) Report of a series of 82 cases of Buruli ulcer from Nigeria treated in Benin, from 2006 to 2016. PLoS Neglected Tropical Diseases 9: 12(3): e0006358. 